\title{
Efficient Binding of Flexible and Redox- active Coenzymes by Oxidoreductases
}

C. Satheesan Babu ${ }^{\dagger, *}$, Carmay $\operatorname{Lim}^{\dagger,+*}$

†Institute of Biomedical Sciences, Academia Sinica, Taipei 11529, Taiwan R. O. C.

Department of Chemistry, National Tsing-Hua University, Hsinchu 300, Taiwan R.O.C.

*E-mail: babu@ibms.sinica.edu.tw or camay@gate.sinica.edu.tw 
Calibration of QM method. The high-resolution $(0.5-\AA)$ X-ray structure of the NAD acid state in the Cambridge Structural Database ${ }^{1}$ (CSD entry 256835.cif) was used to determine an optimal method for treating the coenzyme. The acid state of NAD was geometry optimized using semi-empirical AM12, PM333, $\mathrm{MNDO}^{4}$ and $\mathrm{B} 3-\mathrm{LYP}^{5,6}$ and the $6-31+\mathrm{G}^{*}$ basis set. Comparison of the crystal and fully optimized structures shows that the PM3 method yielded deviations from the experimental geometry that are generally comparable to B3-LYP but much smaller than AM1 or MNDO (see Fig. S1). Hence, the PM3 method seems to be efficient and reliable for QM/MM simulations of NAD. However, the positions of the free energy minima in Figure 2 are independent of the semi-empirical model used.

A

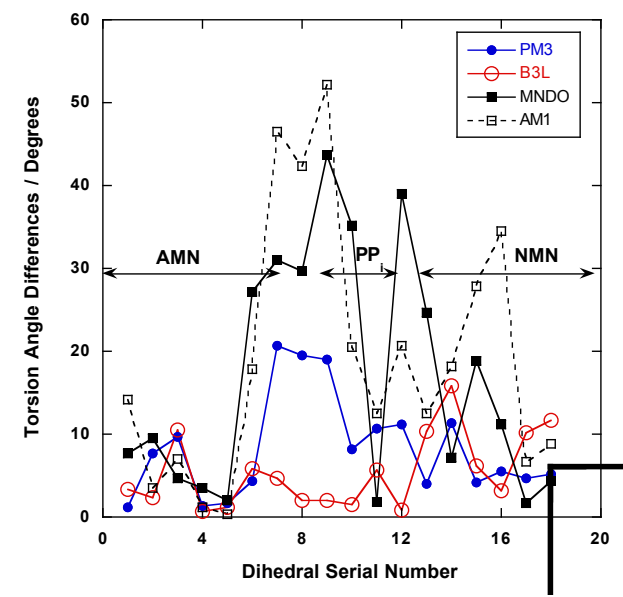

B

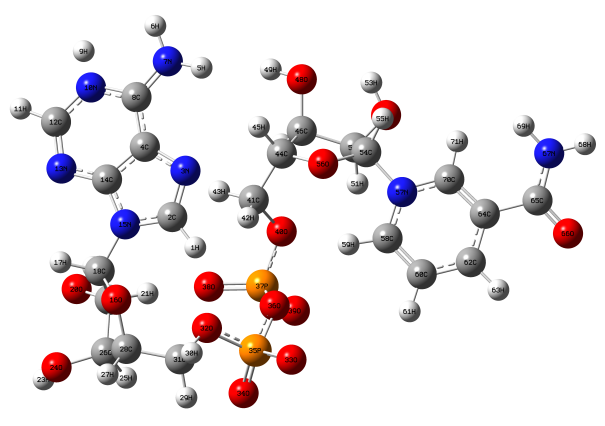

Figure S1. Comparison of the crystal and fully optimized structures of the NAD acid state. (A) Differences between the heavy atom dihedral angles of the NAD acid state in the fully optimized and X-ray (CSD entry 256835.cif) structures as a function of the dihedral angle serial number; the red, blue, black and dashed curves were derived from the NAD acid state fully optimized using B3-LYP, PM3, MNDO and AM1, respectively, and the $6-31+\mathrm{G}^{*}$ basis set. AMN and $\mathrm{NMN}$ denote the adenine and nicotinamide mononucleotide moieties, respectively, whereas PPi denotes the pyrophosphate. (B) The fully optimized structure of the NAD acid state using PM3.

Free energy calculations of $\mathrm{NAD}^{+} / \mathrm{NADH}$ conformations in water. To determine if $\mathrm{NAD}^{+}$and NADH adopt similar solution conformations and if their native solution conformations are compact or

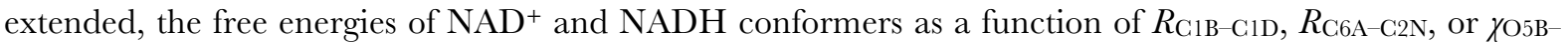
O5D in water were computed using QM/MM simulations with umbrella sampling.

Forcefield. The entire coenzyme was treated using PM3, whereas the water molecules were treated classically using the TIP3P potential ${ }^{7}$, while sodium counter ions employed the CHARMM force field ${ }^{8}$. The water $\mathrm{O}-\mathrm{H}$ bonds were constrained by the SHAKE algorithm ${ }^{9}$. The simulations employed periodic boundary conditions and a nonbond cut-off of $18 \AA$ with the nonbonded interactions smoothly switched to zero from 14 to $17 \AA$.

Simulation Protocol. Molecular dynamics simulations ${ }^{10}$ of $\mathrm{NAD}^{+}$and NADH with the hybrid QM/MM force field were performed using the CHARMM37 program ${ }^{11}$ at $298.15 \mathrm{~K}$ and 1 atm pressure with an integration time step of $1 \mathrm{fs}$. The fully optimized coenzyme $\left(\mathrm{NAD}^{+}\right.$or $\left.\mathrm{NADH}\right)$ was placed at the center of a previously equilibrated $56 \times 56 \times 56 \AA$ cubic box of water molecules of density $1 \mathrm{gm} / \mathrm{cc}$. Water molecules whose oxygen atoms were within $2.5 \AA$ of any cofactor heavy atoms were removed resulting in 5,799 TIP3P water molecules and the cofactor. Sodium counterions were added to neutralize the $\mathrm{NAD}^{+}$or NADH cofactor. The neutral system was equilibrated by initially constraining the cofactor and gradually releasing all constraints. After equilibration, simulations of $\mathrm{NAD}^{+}$and $\mathrm{NADH}$ were performed for $1 \mathrm{~ns}$ each and the coordinates were stored every 0.05 ps for conformational analysis.

Umbrella sampling. The free energies of the cofactor in water as a function of $R_{\mathrm{C} 1 \mathrm{~B}-\mathrm{C} 1 \mathrm{D}}, R_{\mathrm{C} 6 \mathrm{~A}-\mathrm{C} 2 \mathrm{~N}}$, and $\chi_{\mathrm{O} 5 \mathrm{~B}-}$ O5D were obtained by umbrella sampling ${ }^{10}$ and the weighted histogram analysis method ${ }^{12}$ using Grossfield's 
WHAM program. Umbrella forces with a force constant of $10 \mathrm{kcal} . \mathrm{mol}^{-1} / \AA^{2}$ were applied at $\mathrm{R}_{\mathrm{C} 6 \mathrm{~A}-\mathrm{C} 2 \mathrm{~N}} /$ $\mathrm{R}_{\mathrm{C} 1 \mathrm{~B}-\mathrm{C} 1 \mathrm{D}}$ between 6 and $18 \AA$ at an interval of $0.5 \AA$, whereas those with a force constant of $20 \mathrm{kcal}^{-\mathrm{mol}^{-}}$ $1 /$ radian $^{2}$ were applied at $\chi \mathrm{O} 5 \mathrm{~B}-\mathrm{O} 5 \mathrm{D}$ between $-\pi$ and $\pi$ at an interval of 0.25 radians For each of the windows, the system was independently re-equilibrated for $50 \mathrm{ps}$ before a production run of $600 \mathrm{ps}$. The simulation data were divided into three segments of 200 ps. For each 200 ps segment, the free energy at a given reaction coordinate and associated statistical error were computed and the average and standard deviations were obtained from the three sets of data.

Calculation of compact to extended isomerization energy of $\mathbf{N A D}^{+}$and NADH. Compact, syn and extended, anti conformers of $\mathrm{NAD}^{+} / \mathrm{NADH}$ were constrained optimized in water with a polarizable continuum model at the B3-LYP/6-31+G(2d,2p) level using the G09 program ${ }^{13}$. Starting from compact (syn) and extended (anti) conformers from the QM/MM NAD $+/ \mathrm{NADH}$ simulations, the pyrophosphate was frozen, while the rest of the molecule was optimized, and subsequently relaxed keeping the rest of the molecule fixed. The energies of the resulting constrained optimized geometries yielded the compact $\rightarrow$ extended isomerization energy in water; the corresponding isomerization energy in the gas phase was estimated using single-point energy calculations at the B3-LYP/6-31+G(2d,2p) level.

\begin{tabular}{|c|c|c|c|}
\hline \multirow[b]{2}{*}{ Gas-phase: } & $\Delta \mathrm{G}_{\mathrm{gas}}=20 \mathrm{kcal} / \mathrm{mol}$ & \multicolumn{2}{|c|}{$\Delta \mathrm{G}_{\mathrm{gas}}=-23 \mathrm{kcal} / \mathrm{mol}$} \\
\hline & $\mathrm{NAD}^{+}($syn $) \rightarrow \mathrm{NAD}^{+}$(anti) & $\mathrm{NADH}($ syn $) \rightarrow$ & NADH (anti) \\
\hline \multirow{3}{*}{ Solution: } & $\downarrow$ & $\downarrow$ & $\downarrow$ \\
\hline & $\mathrm{NAD}^{+}($syn $) \rightarrow \mathrm{NAD}^{+}$(anti) & $\mathrm{NADH}(\mathrm{syn}) \rightarrow$ & NADH (anti) \\
\hline & $\Delta \mathrm{G}_{\mathrm{sln}}=16 \mathrm{kcal} / \mathrm{mol}$ & $\Delta \mathrm{G}_{\operatorname{sln}}=$ & $-3 \mathrm{kcal} / \mathrm{mol}$ \\
\hline
\end{tabular}

Statistical analyses of NAD+/NADH conformations in enzymes. To determine the nature of the $\mathrm{NAD}^{+}$and $\mathrm{NADH}$ conformations when bound to enzymes, we computed the frequency distributions of $R_{\mathrm{C} 1 \mathrm{~B}-\mathrm{C} 1 \mathrm{D}}, R_{\mathrm{C} 6 \mathrm{~A}-\mathrm{C} 2 \mathrm{~N}}$, and $\chi_{\mathrm{O} 5 \mathrm{~B}-\mathrm{O} 5 \mathrm{D}}$ in oxidoreductase structures containing the ligand NAD (denoting $\mathrm{NAD}^{+}$) or NAI (denoting NADH) ${ }^{14}$ in the Protein Data Bank ${ }^{15}$. Structures with incomplete $\mathrm{NAD}^{+}$or $^{-}$ NADH coordinates were excluded. Homo-oligomeric structures with multiple NAD/NADH were treated as one structure but with the reaction coordinates averaged over all n-mers. This resulted in $382 \mathrm{NAD}^{+}$ and 85 NADH redundant enzyme-bound structures whose PDB codes are listed in Table S1. These structures were used to compute the occurrence frequency of each of the reaction coordinates in enzyme-bound $\mathrm{NAD}^{+}$or NADH structures (see Figures 2 and S2). The free energy profiles for $\mathrm{NAD}^{+}$and NADH computed using PM3, AM1, and MNDO methods all cross around the peak in the frequency distributions (data not shown).

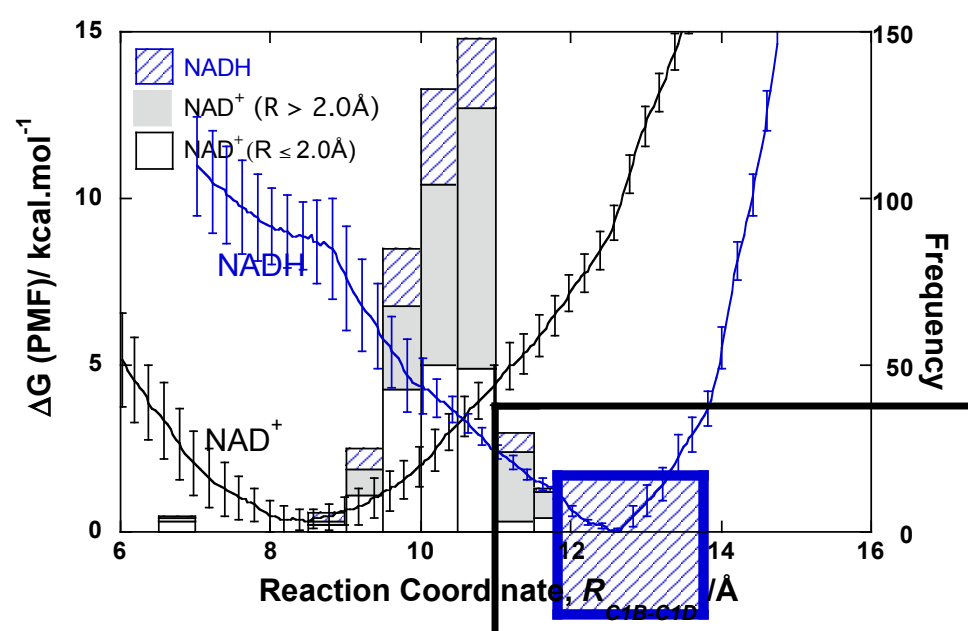

Figure S2. Correlation between $\mathrm{NAD}^{+}$and $\mathrm{NADH}$ conformational free energies in water and their respective frequencies in enzyme-bound X-ray structures. Same as Figure $2 \mathrm{~A}$, except that the $\mathrm{NAD}^{+}$portion of the binding histograms are divided into two regions, viz, one derived from $\leq 2 \AA$ structures (white) and the other from $>2 \AA$ structures (grey). Since the grey and white regions exhibit similar trends, our observations are independent of the resolution of the PDB structures. 
Table S1. $\mathrm{R}_{\mathrm{C} 1 \mathrm{~B}-\mathrm{C} 1 \mathrm{D}}, \mathrm{R}_{\mathrm{C} 6 \mathrm{~A}-\mathrm{C} 2 \mathrm{~N}}$, and $\chi_{\mathrm{O} 5 \mathrm{~B}-\mathrm{O} 5 \mathrm{D}}$ values in $\mathrm{NAD}^{+}$-bound oxidoreductase structures. $^{\text {a }}$

\begin{tabular}{|c|c|c|c|c|}
\hline PDB id & EC Number ${ }^{b}$ & $\mathrm{R}_{\mathrm{C} 1 \mathrm{~B}-\mathrm{C} 1 \mathrm{D}}(\AA)$ & $\mathrm{R}_{\mathrm{C} 6 \mathrm{~A}-\mathrm{C} 2 \mathrm{~N}}(\AA)$ & $\chi_{\mathrm{O} 5 \mathrm{~B}-\mathrm{O} 5 \mathrm{D}}\left({ }^{\circ}\right)$ \\
\hline $1 \mathrm{a} 4 \mathrm{z}$ & 1.2.1.3; & 10.17 & 14.04 & -90.53 \\
\hline $1 \mathrm{a} 5 \mathrm{z}$ & 1.1.1.27; & 9.91 & 13.93 & -95.78 \\
\hline $1 \mathrm{a} 71$ & 1.1.1.1; & 10.44 & 15.05 & -4.13 \\
\hline $1 \mathrm{ad} 3$ & 1.2.1.5; & 10.56 & 13.18 & 89.36 \\
\hline 1ahh & 1.1.1.159; & 10.36 & 13.50 & -30.99 \\
\hline 1axe & 1.1.1.1; & 10.69 & 15.22 & -4.19 \\
\hline $1 \mathrm{axg}$ & 1.1.1.1; & 10.58 & 15.06 & -18.53 \\
\hline $1 b 8 v$ & & 10.01 & 13.87 & -13.34 \\
\hline $1 b d b$ & & 10.20 & 13.90 & 18.55 \\
\hline $1 \mathrm{bmd}$ & 1.1.1.37; & 9.87 & 14.08 & -17.14 \\
\hline $1 \mathrm{bpw}$ & 1.2.1.8; & 10.37 & 14.63 & -122.83 \\
\hline 1bxs & 1.2.1.3 & 10.04 & 12.96 & 136.61 \\
\hline $1 \mathrm{c} 1 \mathrm{x}$ & & 10.44 & 13.94 & -17.00 \\
\hline 1cdo & 1.1.1.1 & 10.77 & 15.21 & -16.65 \\
\hline $1 \mathrm{cwu}$ & 1.3.1.9; & 9.37 & 14.33 & 20.18 \\
\hline $1 \mathrm{~d} 1 \mathrm{~s}$ & 1.1.1.1; & 10.67 & 15.09 & -14.49 \\
\hline $1 \mathrm{~d} 1 \mathrm{t}$ & 1.1.1.1; & 10.59 & 15.03 & -17.00 \\
\hline $1 \mathrm{dbv}$ & 1.2.1.12; & 10.60 & 14.73 & 43.97 \\
\hline $1 \mathrm{dhr}$ & 1.6.99.10; & 9.99 & 14.62 & -37.99 \\
\hline $1 \mathrm{dli}$ & 1.1.1.22; & 11.05 & 14.72 & 36.37 \\
\hline 1dru & 1.3.1.26; & 9.90 & 14.79 & -6.58 \\
\hline $1 d x y$ & 1.1.1.-; & 10.54 & 14.30 & -29.16 \\
\hline $1 \mathrm{ebf}$ & 1.1.1.3; & 10.82 & 15.21 & 10.43 \\
\hline 1 ee 2 & 1.1.1.1 & 10.55 & 15.14 & -8.32 \\
\hline 1 ee9 & 1.5.1.15; & 9.59 & 14.67 & 33.02 \\
\hline 1emd & 1.1.1.37; & 10.10 & 14.15 & 44.13 \\
\hline 1eno & 1.3.1.9; & 9.18 & 14.50 & -93.5 \\
\hline 1eny & & 9.83 & 14.92 & 17.49 \\
\hline 1enz & & 9.88 & 15.01 & -65.66 \\
\hline levj & & 10.62 & 15.02 & 52.82 \\
\hline levz & 1.1.1.8; & 11.04 & 15.81 & 8.94 \\
\hline $1 \mathrm{ez} 4$ & 1.1.1.27; & 9.93 & 15.08 & -9.64 \\
\hline $1 \mathrm{f0y}$ & 1.1.1.35; & 10.53 & 14.31 & 39.86 \\
\hline $1 \mathrm{f} 8 \mathrm{f}$ & 1.1.1.90; & 10.59 & 15.36 & -6.40 \\
\hline $1 \mathrm{f} 8 \mathrm{~g}$ & 1.6.1.1; & 10.06 & 16.07 & 66.57 \\
\hline $1 \mathrm{fk} 8$ & 1.1 .1 .50 & 9.57 & 14.01 & -5.33 \\
\hline 1g6k & 1.1.1.47; & 9.81 & 14.24 & 2.88 \\
\hline 1gad & 1.2.1.12; & 10.77 & 14.99 & 43.08 \\
\hline 1gae & 1.2.1.12; & 11.17 & 15.38 & 47.94 \\
\hline $1 \mathrm{gco}$ & 1.1.1.47; & 9.65 & 14.16 & 2.82 \\
\hline 1gee & 1.1.1.47; & 9.67 & 14.14 & 5.19 \\
\hline 1geg & 1.1.1.5; & 10.13 & 13.86 & -18.46 \\
\hline 1geu & 1.6.4.2; & 12.61 & 17.84 & -175.33 \\
\hline
\end{tabular}




\begin{tabular}{|c|c|c|c|c|}
\hline 1gpd & 1.2.1.12; & 11.36 & 14.14 & 38.66 \\
\hline $1 \mathrm{grb}$ & 1.6.4.2; & 12.37 & 17.48 & -159.19 \\
\hline 1guz & 1.1.1.37; & 10.32 & 14.77 & -20.88 \\
\hline 1gv0 & 1.1.1.37; & 10.31 & 14.68 & -7.35 \\
\hline 1gy 8 & 5.1.3.2; & 9.87 & 14.09 & 3.86 \\
\hline 1gyp & 1.2.1.12; & 10.80 & 14.76 & 62.17 \\
\hline $1 \mathrm{~h} 94$ & 1.1.1.49; & 11.75 & 16.24 & -85.05 \\
\hline 1hdg & 1.2.1.12; & 10.73 & 14.75 & 30.59 \\
\hline 1hdr & 1.6.99.7; & 10.37 & 14.74 & -23.45 \\
\hline 1hdy & 1.1.1.1; & 10.59 & 15.06 & -7.09 \\
\hline $1 \mathrm{hdz}$ & 1.1.1.1; & 10.69 & 15.24 & -23.87 \\
\hline $1 \mathrm{htb}$ & 1.1.1.1; & 10.89 & 15.15 & -27.72 \\
\hline 1hyh & 1.1.1.27; & 10.53 & 15.12 & -10.95 \\
\hline $1 \mathrm{ib} 6$ & 1.1.1.37; & 10.18 & 14.58 & 16.99 \\
\hline $1 \mathrm{il} 0$ & 1.1.1.35; & 10.63 & 14.28 & 44.28 \\
\hline 1iy8 & & 9.72 & 14.24 & -0.97 \\
\hline $1 \mathrm{j} 0 \mathrm{x}$ & 1.2 .1 .12 & 10.84 & 15.19 & 33.62 \\
\hline $1 \mathrm{j} 49$ & 1.1.1.28; & 10.73 & 14.93 & -5.95 \\
\hline $1 j 5 p$ & 1.4.3.16; & 9.84 & 13.92 & -28.74 \\
\hline $1 \mathrm{jq} 5$ & 1.1.1.6; & 12.34 & 16.67 & -141.17 \\
\hline $1 \mathrm{kae}$ & 1.1.1.23; & 12.15 & 16.67 & 127.11 \\
\hline $1 \mathrm{kol}$ & 1.2.1.46; & 10.48 & 14.79 & -34.59 \\
\hline $11 \mathrm{c} 3$ & 1.3.1.24; & 9.35 & 10.06 & 80.98 \\
\hline $11 \mathrm{dm}$ & 1.1.1.27; & 10.18 & 14.89 & -49.21 \\
\hline 1ldn & 1.1.1.27; & 10.67 & 15.04 & -9.27 \\
\hline $11 \mathrm{j} 8$ & 1.1.1.67; & 9.68 & 13.41 & 30.46 \\
\hline 11ld & 1.1.1.27; & 10.51 & 15.46 & 4.23 \\
\hline 11lq & 1.1 .1 .38 & 10.59 & 15.28 & 7.19 \\
\hline 11lu & 1.1.1.1; & 10.64 & 15.19 & -45.22 \\
\hline $11 s j$ & 1.1.1.35; & 10.66 & 14.98 & -8.87 \\
\hline 11so & 1.1.1.35; & 10.79 & 14.77 & 41.98 \\
\hline 11vl & 1.8.1.4; & 10.80 & 14.50 & -163.75 \\
\hline $1 \mathrm{~m} 75$ & 1.1.1.35; & 10.74 & 14.43 & 38.82 \\
\hline $1 \mathrm{~m} 76$ & 1.1.1.35; & 10.56 & 14.29 & 46.54 \\
\hline $1 \mathrm{~m} 9 \mathrm{~h}$ & 1.1.1.-; & 11.71 & 16.66 & -39.06 \\
\hline 1mew & 1.1.1.205; & 12.39 & 15.97 & 73.61 \\
\hline 1mgo & 1.1.1.1; & 10.62 & 15.07 & -5.05 \\
\hline mi3 & 1.1.1.21; & 12.18 & 16.74 & 19.78 \\
\hline $1 \mathrm{mp} 0$ & 1.1.1.1, 1 . & 10.69 & 15.15 & -12.17 \\
\hline lnbo & 1.2.1.13; & 11.00 & 14.77 & 39.26 \\
\hline $1 \mathrm{nfb}$ & 1.1.1.205; & 11.03 & 14.55 & -170.88 \\
\hline $\operatorname{lnff}$ & 1.1.1.53; & 9.70 & 13.89 & -13.33 \\
\hline $1 \mathrm{~nm} 5$ & 1.6.1.2; & 10.07 & 16.84 & 87.66 \\
\hline $1 \mathrm{npt}$ & 1.2.1.12; & 10.91 & 14.83 & 36.95 \\
\hline lnqo & 1.2.1.12; & 10.93 & 14.85 & 35.97 \\
\hline 1002 & 1.2.1.3; & 10.04 & 12.93 & 126.48 \\
\hline
\end{tabular}




\begin{tabular}{|c|c|c|c|c|}
\hline 1004 & 1.2.1.3; & 10.24 & 14.50 & -94.28 \\
\hline $106 z$ & 1.1.1.37; & 10.35 & 14.92 & -6.97 \\
\hline $109 j$ & 1.2.1.3; & 10.45 & 14.62 & -75.62 \\
\hline $10 \mathrm{~s} 4$ & 1.1.1.27; & 10.43 & 14.79 & -7.37 \\
\hline 1omo & 1.4.1.1; & 10.30 & 14.25 & -42.23 \\
\hline $1 p j c$ & 1.4.1.1; & 10.68 & 15.57 & 23.02 \\
\hline $1 \mathrm{pl} 8$ & 1.1.1.14; & 10.40 & 14.79 & -11.22 \\
\hline $1 \mathrm{pzh}$ & 1.1.1.27; & 10.28 & 14.74 & -17.50 \\
\hline 1qlh & 1.1.1.1; & 11.47 & 15.88 & -36.36 \\
\hline $1 \mathrm{qr} 6$ & 1.1.1.39; & 10.05 & 13.92 & 79.44 \\
\hline 1qsg & 1.3.1.9; & 9.39 & 14.61 & -7.51 \\
\hline 1qv7 & 1.1.1.1; & 10.65 & 15.22 & -10.36 \\
\hline $1 \mathrm{r} 37$ & 1.1.1.1; & 10.67 & 15.14 & 3.53 \\
\hline 1rwb & 1.1.1.47; & 9.93 & 14.11 & 2.27 \\
\hline 1rz1 & & 6.75 & 3.87 & -1.22 \\
\hline $1 \mathrm{~s} 20$ & 1.1.1.-; & 11.14 & 17.25 & 31.73 \\
\hline 1 sby & 1.1 .1 .1 & 9.03 & 13.95 & -12.42 \\
\hline $1 \mathrm{sc} 6$ & 1.1.1.95; & 10.48 & 14.35 & -33.10 \\
\hline $1 \mathrm{sm} 9$ & 1.1.1.21; & 12.26 & 16.73 & -39.70 \\
\hline 1sow & 1.1.1.27; & 10.35 & 14.88 & -17.28 \\
\hline $1 \mathrm{t} 2 \mathrm{~d}$ & 1.1.1.27; & 10.36 & 14.65 & -10.39 \\
\hline $1 \mathrm{t} 2 \mathrm{f}$ & 1.1.1.27; & 10.12 & 14.68 & -4.21 \\
\hline $1 \mathrm{t} 90$ & 1.2.1.27; & 10.15 & 14.51 & -79.21 \\
\hline $1 \mathrm{u} 3 \mathrm{t}$ & 1.1.1.1; & 10.66 & 15.20 & -36.73 \\
\hline $1 \mathrm{u} 3 \mathrm{u}$ & 1.1.1.1; & 10.85 & 15.20 & -7.53 \\
\hline $1 \mathrm{u} 3 \mathrm{w}$ & 1.1.1.1; & 10.71 & 14.98 & -8.26 \\
\hline $1 \mathrm{u} 7 \mathrm{t}$ & 1.1.1.35; & 9.73 & 14.14 & -16.85 \\
\hline $1 \mathrm{u} 8 \mathrm{f}$ & 1.2.1.12; & 10.91 & 14.92 & 30.89 \\
\hline 1ur5 & 1.1.1.37; & 10.60 & 15.25 & -5.88 \\
\hline luxg & 1.1.1.37; & 10.71 & 15.08 & -2.00 \\
\hline 1uxj & 1.1.1.37; & 10.76 & 15.10 & -0.13 \\
\hline luxk & 1.1.1.37; & 10.74 & 15.03 & -4.14 \\
\hline luxt & 1.2.1.9; & 10.57 & 14.72 & -128.34 \\
\hline 1v91 & 1.4.1.2 & 10.53 & 14.47 & 6.44 \\
\hline 1vbi & 1.1.1.27; & 11.57 & 17.98 & -27.66 \\
\hline $1 \mathrm{vc} 2$ & 1.2.1.12; & 10.30 & 14.49 & -38.08 \\
\hline 1vi2 & 1.1.1.25; & 10.10 & 15.05 & 24.87 \\
\hline $1 \mathrm{vm} 6$ & 1.3.1.26; & 10.08 & 14.64 & 10.78 \\
\hline $1 \mathrm{vsv}$ & 1.2.1.12; & 10.82 & 14.97 & 4.80 \\
\hline $1 w p q$ & 1.1.1.8; & 11.39 & 15.60 & -163.09 \\
\hline 1wwk & 1.1.1.95; & 10.84 & 15.05 & -42.39 \\
\hline $1 \mathrm{x} 0 \mathrm{x}$ & 1.1.1.8; & 11.16 & 15.75 & -151.65 \\
\hline $1 \times 14$ & 1.6.1.2; & 10.82 & 17.07 & 41.40 \\
\hline $1 \times 1 t$ & 1.1.1.30; & 9.83 & 14.37 & 44.06 \\
\hline $1 \times 31$ & 1.5.3.1; & 11.85 & 17.59 & -110.43 \\
\hline 1yba & 1.1.1.95; & 10.68 & 14.80 & -21.45 \\
\hline
\end{tabular}




\begin{tabular}{|c|c|c|c|c|}
\hline 1ye6 & 1.1.1.-; & 12.23 & 16.67 & 52.59 \\
\hline 1ywg & 1.2.1.12; & 10.82 & 15.47 & 84.44 \\
\hline $1 z 2 \mathrm{i}$ & & 11.59 & 18.24 & 36.14 \\
\hline $1 z 9 a$ & 1.1.1.-; & 12.15 & 16.62 & 48.50 \\
\hline 1zem & 1.1.1.9; & 9.65 & 14.08 & -17.28 \\
\hline $1 z j z$ & 1.1.1.2; & 8.66 & 13.42 & -86.62 \\
\hline 2ag5 & & 10.41 & 14.26 & -26.44 \\
\hline $2 \mathrm{~b} 4 \mathrm{r}$ & 1.2.1.12; & 10.77 & 14.86 & 37.20 \\
\hline 2bjk & 1.5.1.12; & 10.40 & 14.48 & -98.39 \\
\hline $2 \mathrm{bkj}$ & 1.6.99.-; & 5.68 & 3.89 & -10.48 \\
\hline $2 \mathrm{bl} 4$ & 1.1.1.77; & 12.42 & 16.80 & -0.06 \\
\hline $2 \mathrm{cfc}$ & 1.1 .1 .268 & 9.08 & 13.69 & 19.36 \\
\hline $2 \mathrm{czc}$ & 1.2.1.59; & 10.66 & 14.36 & 42.64 \\
\hline $2 \mathrm{~d} 1 \mathrm{y}$ & & 9.90 & 14.50 & -63.23 \\
\hline 2d37 & 1.6.8.-; & 6.76 & 4.22 & 49.35 \\
\hline $2 \mathrm{~d} 4 \mathrm{e}$ & 1.2.1.60; & 10.52 & 15.03 & 32.87 \\
\hline $2 \mathrm{~d} 4 \mathrm{v}$ & & 10.30 & 11.16 & 11.82 \\
\hline $2 \mathrm{~d} 8 \mathrm{a}$ & 1.1.1.103; & 10.29 & 14.95 & -69.48 \\
\hline $2 \mathrm{dc} 1$ & 1.4.1.21; & 9.92 & 13.97 & -26.40 \\
\hline $2 \mathrm{dfd}$ & 1.1.1.37; & 10.33 & 14.8206 & -1.0252 \\
\hline $2 \mathrm{dph}$ & 1.2 .99 .4 & 10.70 & 14.73 & -16.87 \\
\hline $2 \mathrm{dvm}$ & 1.1.1.38; & 9.93 & 14.00 & 10.09 \\
\hline 2ed4 & 1.6.8.-; & 6.72 & 4.48 & 41.73 \\
\hline 2eer & 1.1.1.1; & 10.53 & 14.87 & -21.09 \\
\hline 2ejv & 1.1.1.103; & 10.50 & 15.57 & -58.35 \\
\hline 2ekl & 1.1.1.95; & 10.14 & 14.78 & -5.45 \\
\hline 2ekp & 1.1.1.125; & 10.14 & 14.45 & 20.36 \\
\hline 2ep7 & 1.2.1.12; & 10.74 & 14.76 & -14.18 \\
\hline 2eq7 & 2.3.1.61; & 12.05 & 17.71 & -156.67 \\
\hline $2 \mathrm{fm} 3$ & 1.1.1.27; & 10.71 & 15.04 & -18.36 \\
\hline $2 \mathrm{fsv}$ & 1.6.1.2; & 9.95 & 15.11 & 67.90 \\
\hline $2 \mathrm{fzw}$ & 1.1.1.1, 1 . & 10.33 & 14.15 & -17.49 \\
\hline $2 \mathrm{~g} 5 \mathrm{c}$ & 1.3.1.12; & 10.39 & 14.17 & 13.23 \\
\hline $2 \mathrm{~g} 76$ & 1.1.1.95; & 10.31 & 14.96 & -15.07 \\
\hline $2 \mathrm{~g} 82$ & 1.2.1.12; & 10.73 & 14.74 & 22.92 \\
\hline $2 g 8 y$ & & 10.88 & 17.60 & -10.40 \\
\hline 2gag & & 11.94 & 17.53 & -105.78 \\
\hline $2 \mathrm{gdz}$ & 1.1.1.141; & 9.88 & 13.88 & 10.51 \\
\hline $2 \mathrm{gsd}$ & 1.2.1.43; & 9.99 & 14.59 & -7.45 \\
\hline 2hae & & 9.46 & 13.59 & -20.00 \\
\hline 2hdh & 1.1.1.35; & 10.69 & 14.55 & 38.29 \\
\hline $2 \mathrm{hsd}$ & 1.1.1.53; & 9.68 & 13.88 & -35.35 \\
\hline $2 \mathrm{i} 9 \mathrm{p}$ & 1.1.1.31; & 11.45 & 15.45 & -19.48 \\
\hline $2 i z z$ & 1.5.1.2; & 10.65 & 14.90 & 23.89 \\
\hline 2jg7 & & 10.28 & 14.61 & -82.64 \\
\hline $2 \mathrm{jhf}$ & 1.1.1.1; & 10.82 & 15.34 & -2.46 \\
\hline
\end{tabular}




\begin{tabular}{|c|c|c|c|c|}
\hline 2jjy & 1.3.1.9; & 9.68 & 14.84 & -107.76 \\
\hline 2 nad & 1.2.1.2; & 9.97 & 14.72 & -0.84 \\
\hline $2 n p x$ & 1.11.1.1; & 11.80 & 17.28 & -165.99 \\
\hline 2023 & 1.1.1.178; & 9.84 & 14.01 & -3.70 \\
\hline $202 y$ & 1.3.1.9; & 9.96 & 14.25 & 21.15 \\
\hline $204 c$ & 1.1.1.290; & 10.36 & 14.77 & -15.25 \\
\hline 2ome & & 10.04 & 14.26 & -13.00 \\
\hline 2onm & 1.2.1.3; & 9.89 & 12.97 & 152.51 \\
\hline 2onp & 1.2.1.3; & 10.12 & 13.03 & 161.65 \\
\hline $200 \mathrm{~s}$ & 1.3.1.9; & 9.81 & 14.36 & 22.97 \\
\hline $2 \mathrm{pd} 4$ & 1.3.1.9; & 9.52 & 14.56 & -39.21 \\
\hline $2 \mathrm{pd} 6$ & 1.1.1.62; & 9.93 & 13.95 & -4.63 \\
\hline 2pla & & 11.23 & 15.66 & -146.46 \\
\hline $2 q 2 v$ & 1.1.1.30; & 9.85 & 13.97 & 0.36 \\
\hline $2 \mathrm{qg} 4$ & 1.1.1.22; & 11.11 & 15.24 & 30.57 \\
\hline $2 \mathrm{rab}$ & & 13.16 & 19.00 & 164.63 \\
\hline $2 \mathrm{v} 7 \mathrm{p}$ & 1.1.1.27; & 10.37 & 15.10 & 3.60 \\
\hline $2 v h x$ & 1.4.1.1; & 10.80 & 15.45 & 10.10 \\
\hline $2 v y n$ & 1.2 .1 .12 & 10.83 & 14.85 & 26.90 \\
\hline $2 w 21$ & & 10.29 & 14.17 & -47.01 \\
\hline $2 \mathrm{wsb}$ & 1.1.1.16; & 10.10 & 13.91 & -11.41 \\
\hline 2 wyv & 1.3.1.10; & 9.66 & 15.05 & -11.55 \\
\hline $2 \times 06$ & 1.1.1.272; & 11.30 & 18.12 & 35.7572 \\
\hline $2 \times 0 n$ & 1.2 .1 .12 & 10.93 & 15.30 & 39.14 \\
\hline $2 x x j$ & 1.1.1.27; & 10.64 & 15.20 & 19.04 \\
\hline $2 y q 5$ & & 10.56 & 14.96 & -8.34 \\
\hline $2 y v g$ & 1.18.1.2; & 12.10 & 16.47 & -170.25 \\
\hline $2 y y 7$ & 1.1 .1 .103 & 10.69 & 14.73 & -27.12 \\
\hline $2 \mathrm{ztm}$ & 1.1.1.30; & 10.05 & 14.38 & 7.07 \\
\hline $2 z t u$ & 1.1.1.30; & 10.20 & 14.67 & 2.63 \\
\hline $3 a 28$ & & 9.94 & 14.02 & -3.89 \\
\hline $3 a 4 v$ & 1.1.1.103; & 10.16 & 14.38 & -29.58 \\
\hline 3abi & 1.4.1.18; & 10.53 & 16.02 & -73.95 \\
\hline 3ajr & 1.1.1.103; & 10.17 & 14.39 & -34.40 \\
\hline $3 \mathrm{am} 4$ & 1.3.1.9; & 9.84 & 14.27 & -9.48 \\
\hline $3 a m 5$ & 1.3.1.9; & 9.92 & 14.38 & 38.08 \\
\hline $3 b 1 f$ & 1.3.1.12; & 10.10 & 14.17 & 14.42 \\
\hline $3 b 1 j$ & 1.2.1.13; & 10.74 & 14.89 & 46.69 \\
\hline $3 b 4 w$ & 1.2.1.-; & 10.47 & 14.54 & -84.91 \\
\hline $3 c 7 a$ & 1.5.1.11; & 11.66 & 15.70 & -65.96 \\
\hline 3 cea & 1.1.1.18; & 10.55 & 15.48 & 6.41 \\
\hline $3 \operatorname{cgd}$ & & 12.27 & 17.17 & -164.64 \\
\hline 3 cif & 1.2.1.12; & 10.83 & 14.87 & 30.33 \\
\hline $3 \mathrm{cmc}$ & 1.2.1.12; & 10.91 & 14.53 & 31.24 \\
\hline $3 \cos$ & 1.1.1.1; & 10.37 & 14.65 & -15.59 \\
\hline $3 \mathrm{cps}$ & & 10.70 & 14.80 & 25.96 \\
\hline
\end{tabular}




\begin{tabular}{|c|c|c|c|c|}
\hline $3 d 4 p$ & 1.1.1.27; & 10.48 & 15.09 & 1.16 \\
\hline $3 \mathrm{dbv}$ & 1.2.1.12 & 10.75 & 14.99 & 43.01 \\
\hline $3 \mathrm{doc}$ & & 11.02 & 14.88 & 16.86 \\
\hline $3 \mathrm{e} 18$ & & 10.68 & 15.26 & 45.13 \\
\hline $3 e c 7$ & & 10.55 & 15.52 & 36.68 \\
\hline $3 \mathrm{efv}$ & 1.2.1.-; & 10.22 & 14.71 & -77.99 \\
\hline $3 f 4 b$ & 1.3.1.9; & 9.96 & 14.29 & 19.16 \\
\hline $3 g d 4$ & & 11.02 & 16.27 & 177.20 \\
\hline 3 geg & & 10.03 & 13.63 & 14.60 \\
\hline $3 \mathrm{gfb}$ & 1.1.1.103; & 10.64 & 15.08 & -50.81 \\
\hline 3gnq & 1.2.1.-; & 11.01 & 14.78 & 30.21 \\
\hline 3gpd & 1.2.1.12; & 11.76 & 16.17 & 8.71 \\
\hline 3gvh & 1.1.1.37; & 10.57 & 14.83 & -16.93 \\
\hline $3 \mathrm{~h} 3 \mathrm{j}$ & 1.1.1.27; & 10.30 & 14.94 & -4.01 \\
\hline $3 \mathrm{~h} 9 \mathrm{e}$ & 1.2.1.12; & 10.85 & 14.62 & 33.55 \\
\hline 3had & 1.1.1.35; & 10.56 & 14.50 & 24.20 \\
\hline 3haz & $1.5 .99 .8,1$ & 10.33 & 14.68 & -101.09 \\
\hline 3hdh & 1.1 .1 .35 & 10.76 & 14.65 & 41.74 \\
\hline 3hja & 1.2.1.12; & 10.89 & 14.77 & 22.12 \\
\hline $3 \mathrm{hl0}$ & & 11.92 & 15.72 & -131.95 \\
\hline 3hq4 & 1.2.1.12; & 10.76 & 14.75 & 23.47 \\
\hline $3 \mathrm{i} 0 \mathrm{p}$ & & 11.09 & 17.66 & 2.40 \\
\hline 3ids & 1.2.1.12; & 11.02 & 14.88 & 30.36 \\
\hline $3 i j r$ & & 9.71 & 14.53 & 21.48 \\
\hline 3iwk & 1.2.1.19; & 10.19 & 12.44 & 46.94 \\
\hline 3jsa & & 10.49 & 14.68 & -9.78 \\
\hline $3 \mathrm{ju} 8$ & 1.2.1.71; & 10.58 & 14.91 & -90.59 \\
\hline 3jv7 & 1.1.1.1; & 10.40 & 14.26 & -12.73 \\
\hline 3јуо & $1.1 .1 .24,1$ & 10.65 & 15.07 & 1.81 \\
\hline 3jzd & & 11.97 & 15.87 & -135.2 \\
\hline $3 \mathrm{k} 88$ & & 6.72 & 4.32 & 21.63 \\
\hline $3 \mathrm{~kb} 6$ & 1.1.1.28; & 10.46 & 15.07 & -0.26 \\
\hline $3 \mathrm{kft}$ & & 10.94 & 14.59 & 113.37 \\
\hline $3 \mathrm{ksd}$ & 1.2.1.12; & 10.78 & 14.67 & 43.60 \\
\hline $310 \mathrm{~d}$ & 1.2.1.12; & 11.26 & 15.26 & 36.05 \\
\hline $314 s$ & 1.2.1.12; & 10.76 & 14.70 & 33.57 \\
\hline $31 \mathrm{c} 1$ & 1.2.1.12; & 10.75 & 14.63 & 36.50 \\
\hline $31 \mathrm{dh}$ & 1.1.1.27; & 9.24 & 14.13 & -13.83 \\
\hline $3 \ln 3$ & & 12.57 & 17.71 & 17.71 \\
\hline $31 t 0$ & 1.3.1.9; & 9.86 & 14.33 & 36.33 \\
\hline $3 \operatorname{lvf}$ & 1.2.1.12; & 10.80 & 14.74 & 35.68 \\
\hline $3 \mathrm{~m} 2 \mathrm{t}$ & & 10.62 & 15.79 & 29.25 \\
\hline $3 \mathrm{~m} 6 \mathrm{i}$ & 1.1.1.12; & 10.47 & 15.01 & -33.75 \\
\hline $3 n 7 u$ & 1.2.1.2; & 10.59 & 15.42 & -8.12 \\
\hline $3 n 82$ & 1.2.1.3; & 10.00 & 12.93 & 124.60 \\
\hline $3 \mathrm{nrc}$ & 1.3.1.9; & 9.20 & 14.16 & 2.82 \\
\hline
\end{tabular}




\begin{tabular}{|c|c|c|c|c|}
\hline $3 n t 2$ & 1.1.1.18; & 10.74 & 14.77 & -1.17 \\
\hline $3 n t q$ & 1.1.1.18; & 10.91 & 14.87 & -14.98 \\
\hline $309 z$ & & 10.39 & 15.02 & 59.42 \\
\hline 3 oa2 & & 10.65 & 15.49 & 52.83 \\
\hline 3oet & 1.1.1.290; & 10.35 & 14.71 & -33.16 \\
\hline 3oey & 1.3.1.9; & 9.55 & 14.57 & 11.16 \\
\hline 3 of 2 & 1.3.1.9; & 9.50 & 14.58 & 10.13 \\
\hline 3oig & 1.3.1.9; & 9.33 & 14.48 & 14.94 \\
\hline 3ojo & & 11.02 & 14.32 & -3.91 \\
\hline 3orf & 1.5.1.34; & 10.07 & 13.95 & -21.44 \\
\hline $30 \times 4$ & 1.1.1.1; & 12.19 & 16.67 & -171.31 \\
\hline $3 p 2 o$ & 1.5.1.5, 3 & 10.36 & 14.92 & -31.68 \\
\hline $3 p g x$ & & 9.49 & 13.69 & -19.61 \\
\hline 3pjd & 1.3.1.9; & 9.39 & 14.45 & 46.29 \\
\hline 3 pje & 1.3.1.9; & 9.34 & 14.32 & 43.04 \\
\hline 3pjf & 1.3.1.9; & 9.61 & 14.89 & 30.54 \\
\hline 3pqd & 1.1.1.27; & 10.23 & 15.58 & 6.22 \\
\hline $3 p q f$ & 1.1.1.27; & 9.47 & 13.79 & -52.05 \\
\hline $3 \mathrm{ptz}$ & 1.1.1.22; & 10.59 & 12.36 & -58.27 \\
\hline $3 p x x$ & 1.1.1.275; & 9.62 & 13.56 & -14.95 \\
\hline 3pym & 1.2 .1 .12 & 10.75 & 14.71 & 41.545 \\
\hline $3 q 3 c$ & 1.1.1.276; & 11.53 & 15.67 & -17.47 \\
\hline $3 q 6 i$ & 1.1.1.100; & 10.08 & 14.29 & -51.99 \\
\hline $3 q j 5$ & 1.1.1.1, 1 . & 10.71 & 15.15 & -9.55 \\
\hline $3 q v 1$ & 1.2.1.13; & 10.71 & 14.84 & 39.02 \\
\hline $3 r 3 s$ & & 9.30 & 14.22 & 29.42 \\
\hline $3 \mathrm{rf} 7$ & & 12.18 & 16.36 & -162.11 \\
\hline $3 \mathrm{rj} 5$ & 1.1.1.1; & 8.95 & 13.90 & -13.19 \\
\hline 3rwb & 1.1.1.107; & 10.03 & 13.86 & -14.20 \\
\hline $3 \mathrm{~s} 2 \mathrm{e}$ & & 10.17 & 14.65 & 2.37 \\
\hline $3 \mathrm{~s} 55$ & & 9.65 & 13.58 & -6.35 \\
\hline $3 \mathrm{sth}$ & 1.2.1.12; & 10.86 & 14.91 & 17.84 \\
\hline $3 \mathrm{sx} 2$ & & 9.72 & 13.70 & -23.36 \\
\hline $3 \mathrm{t} 4 \mathrm{e}$ & 1.1.1.282; & 10.07 & 15.04 & 25.25 \\
\hline $3 \mathrm{t} 7 \mathrm{c}$ & 1.1.1.275; & 9.79 & 13.76 & -27.55 \\
\hline $3 \operatorname{tnl}$ & 1.1.1.25; & 9.86 & 14.95 & 19.78 \\
\hline 3 tsc & & 9.58 & 13.47 & -27.58 \\
\hline 3uha & 1.5.1.7; & 10.68 & 14.87 & -6.84 \\
\hline 3uko & 1.1.1.1, 1 . & 10.87 & 15.17 & 0.82 \\
\hline 3uve & 1.1.1.275; & 9.55 & 13.66 & -22.44 \\
\hline 3uxy & & 9.26 & 14.36 & -46.10 \\
\hline $3 v 1 u$ & 1.1.1.100; & 10.05 & 14.08 & 5.39 \\
\hline $3 v 1 y$ & 1.2.1.12; & 10.91 & 14.99 & 36.29 \\
\hline $3 v 91$ & 1.5.1.12; & 10.28 & 14.86 & -80.25 \\
\hline $3 v p h$ & 1.1.1.27; & 10.42 & 15.21 & -0.78 \\
\hline $3 w 2 g$ & 1.6.2.2; & 9.58 & 13.58 & 13.40 \\
\hline
\end{tabular}




\begin{tabular}{|c|c|c|c|c|}
\hline $3 \mathrm{w} 8 \mathrm{e}$ & 1.1.1.30; & 9.94 & 14.08 & -6.88 \\
\hline $3 w d s$ & & 9.58 & 13.86 & -11.10 \\
\hline 3wmx & 1.-.-.-; & 10.55 & 14.62 & -40.77 \\
\hline $3 z v 6$ & 1.3.1.56; & 10.08 & 14.10 & 13.76 \\
\hline $3 z w c$ & $4.2 .1 .17,5$ & 10.74 & 14.75 & 53.65 \\
\hline $4 \mathrm{a} 0 \mathrm{~m}$ & 1.2.1.8; & 10.54 & 14.65 & -102.10 \\
\hline $4 a 7 p$ & 1.1.1.22; & 10.71 & 14.87 & -18.53 \\
\hline $4 b 79$ & & 9.78 & 14.53 & -8.16 \\
\hline 4bgi & 1.3.1.9; & 9.81 & 15.06 & -52.96 \\
\hline 4boy & 1.2.1.13; & 10.88 & 14.79 & 39.93 \\
\hline $4 c 3 s$ & 1.2.1.10; & 9.93 & 13.53 & -65.01 \\
\hline $4 \mathrm{~d} 19$ & 1.1.1.1; & 10.76 & 15.22 & 1.53 \\
\hline 4dyd & & 10.58 & 14.40 & -65.53 \\
\hline $4 e 5 n$ & & 10.52 & 15.04 & -34.35 \\
\hline $4 e 5 p$ & & 10.72 & 15.34 & -24.44 \\
\hline $4 \mathrm{ebf}$ & & 10.62 & 15.26 & -35.46 \\
\hline 4emi & & 12.01 & 16.81 & -169.16 \\
\hline 4euf & 1.3.1.-; & 11.14 & 15.82 & -27.80 \\
\hline $4 \mathrm{f} 3 \mathrm{x}$ & 1.2.1.-; & 10.21 & 12.64 & 138.59 \\
\hline $4 \mathrm{f} 7 \mathrm{i}$ & 1.1.1.85; & 10.22 & 11.37 & 112.99 \\
\hline $4 \mathrm{fbg}$ & 1.3.1.44; & 10.89 & 14.97 & -43.00 \\
\hline $4 \mathrm{fn} 4$ & 1.-.-.-; & 9.98 & 13.75 & -6.45 \\
\hline 4gap & 1.6.5.9; & 12.47 & 19.14 & 157.11 \\
\hline $4 \operatorname{gh} 5$ & & 10.11 & 13.67 & -28.89 \\
\hline $4 \mathrm{gkv}$ & 1.1.1.1; & 10.26 & 14.51 & -5.52 \\
\hline 4glo & 1.1.1.100; & 19.59 & 22.26 & 7.88 \\
\hline 4go4 & 1.2.1.3; & 10.19 & 14.74 & -33.75 \\
\hline $4 \mathrm{ili}$ & 1.1.1.37; & 9.96 & 14.33 & -13.60 \\
\hline $4 \mathrm{i} 1 \mathrm{w}$ & & 10.47 & 14.74 & -91.68 \\
\hline $4 i 4 b$ & 1.1.1.88; & 12.72 & 16.15 & -174.81 \\
\hline $4 i 8 p$ & 1.2.1.19; & 10.27 & 14.48 & -100.37 \\
\hline $4 i 8 q$ & & 10.61 & 14.90 & -99.10 \\
\hline $4 i d 9$ & & 9.75 & 14.21 & -35.70 \\
\hline 4iin & 1.1.1.100; & 9.56 & 13.88 & -23.41 \\
\hline 4 is 3 & & 9.63 & 13.48 & -21.73 \\
\hline $4 \mathrm{j} 1 \mathrm{t}$ & 1.6.1.2; & 8.76 & 14.33 & 1.91 \\
\hline $4 \mathrm{jlw}$ & & 10.26 & 14.55 & -29.99 \\
\hline $4 \mathrm{k} 28$ & & 10.22 & 14.60 & 16.41 \\
\hline $4 \mathrm{k} 9 \mathrm{~d}$ & 1.2.1.12; & 10.92 & 15.03 & 28.75 \\
\hline $4 \mathrm{kqx}$ & 1.1.1.86; & 11.48 & 14.76 & 18.97 \\
\hline 4kug & 1.1.1.157; & 10.47 & 14.23 & 38.57 \\
\hline $410 q$ & 1.1.1.-; & 10.95 & 15.24 & 11.84 \\
\hline 4120 & 1.2.1.5; & 10.82 & 15.59 & -73.79 \\
\hline 4lcj & & 10.10 & 14.39 & -12.82 \\
\hline 4lrs & 1.2.1.10; & 10.55 & 14.31 & 35.46 \\
\hline $41 \mathrm{sm}$ & 1.2.1.12; & 11.00 & 15.02 & 33.78 \\
\hline
\end{tabular}




$\begin{array}{llrrr}\text { 4m89 } & 1.3 .1 .9 ; & 9.21 & 14.32 & 14.12 \\ \text { 4mp8 } & & 10.46 & 13.86 & -2.65 \\ \text { 4mpy } & 1.2 .1 .8 ; & 10.26 & 13.41 & 165.97 \\ \text { 4nbt } & 1.1 .1 .100 ; & 9.63 & 14.05 & -21.28 \\ \text { 4nbw } & & 10.35 & 14.41 & 1.80 \\ \text { 4nk5 } & 1.3 .1 .9 ; & 9.71 & 14.57 & -58.93 \\ \text { 4nu5 } & 1.20 .1 .1 ; & 10.45 & 15.05 & -32.30 \\ \text { 4nu6 } & 1.20 .1 .1 ; & 10.17 & 15.05 & -37.37 \\ \text { 4o1m } & 1.3 .1 .9 ; & 9.64 & 14.29 & -61.74 \\ \text { 4ohu } & 1.3 .1 .9 ; & 9.39 & 14.53 & 23.38 \\ \text { 4p8r } & 1.2 .1 .12 ; & 11.16 & 15.02 & 21.06 \\ \text { 4plf } & & 10.29 & 14.84 & -8.18 \\ \text { 4prl } & 1.1 .1 .290 ; & 10.29 & 14.96 & -72.16 \\ \text { 4q3c } & 1.4 .1 .-; & 10.47 & 15.11 & -2.68 \\ \text { 4qn2 } & 1.2 .1 .8 ; & 10.54 & 14.97 & -59.96 \\ \text { 4tkm } & & 10.24 & 13.99 & -91.73 \\ \text { 5mdh } & 1.1 .1 .37 ; & 10.19 & 14.22 & 49.83 \\ \text { 9ldt } & 1.1 .1 .27 ; & 10.24 & 14.84 & 8.00\end{array}$

${ }^{\mathrm{a} C}$ Compact $\mathrm{NAD}^{+}$structures with $R_{\mathrm{C} 1 \mathrm{~B}-\mathrm{C} 1 \mathrm{D}}$ and $R_{\mathrm{C} 6 \mathrm{~A}-\mathrm{C} 2 \mathrm{~N}}<7 \AA$ are highlighted in bold. ${ }^{\mathrm{b}} \mathrm{A}$ blank in the $\mathrm{EC}$ column indicates the $\mathrm{EC}$ number is uncertain. 
Table S2. $\mathrm{R}_{\mathrm{C} 1 \mathrm{~B}-\mathrm{C} 1 \mathrm{D}}, \mathrm{R}_{\mathrm{C} 6 \mathrm{~A}-\mathrm{C} 2 \mathrm{~N}}$, and $\chi_{\mathrm{O} 5 \mathrm{~B}-\mathrm{O} 5 \mathrm{D}}$ values in NADH -bound oxidoreductase structures.

\begin{tabular}{|c|c|c|c|c|}
\hline PDB id & $\mathrm{EC} \#^{\mathrm{a}}$ & $\mathrm{R}_{\mathrm{C} 1 \mathrm{~B}-\mathrm{C} 1 \mathrm{D}}(\AA)$ & $\mathrm{R}_{\mathrm{C} 6 \mathrm{~A}-\mathrm{C} 2 \mathrm{~N}}(\AA)$ & $\mathrm{X}_{\mathrm{O} 5 \mathrm{~B}-\mathrm{O} 5 \mathrm{D}}\left({ }^{\circ}\right)$ \\
\hline $1 \mathrm{p} 1 \mathrm{r}$ & 1.1.1.1 & 10.63 & 15.12 & -6.65 \\
\hline $2 \mathrm{j} 61$ & 1.2.1.3; & 10.22 & 14.30 & -86.73 \\
\hline larz & 1.3.1.26; & 10.55 & 14.24 & -19.55 \\
\hline $1 \mathrm{cld}$ & & 10.41 & 14.25 & -12.78 \\
\hline $1 \mathrm{dlj}$ & 1.1.1.22; & 11.02 & 14.72 & 33.62 \\
\hline $1 \mathrm{e} 3 \mathrm{i}$ & 1.1.1.1; & 10.69 & 15.04 & -32.34 \\
\hline $1 \mathrm{f} 17$ & 1.1.1.35; & 10.66 & 14.56 & 53.25 \\
\hline $1 \mathrm{fmc}$ & 1.1.1.159; & 10.04 & 13.44 & 15.63 \\
\hline $1 \mathrm{i} 0 \mathrm{z}$ & 1.1.1.27; & 10.38 & 14.85 & 0.76 \\
\hline $117 \mathrm{e}$ & 1.6.1.1; & 9.99 & 17.08 & -53.25 \\
\hline $11 d g$ & 1.1.1.27; & 10.40 & 14.72 & -9.54 \\
\hline $1 \mathrm{mg} 5$ & 1.1.1.1 & 8.88 & 13.83 & -10.11 \\
\hline $\ln 2 \mathrm{~s}$ & 1.1.1.133; & 10.09 & 14.27 & -30.59 \\
\hline $\operatorname{lnfq}$ & 1.1.1.53; & 9.90 & 14.01 & -15.05 \\
\hline 1nzw & 1.2.1.3; & 10.07 & 13.00 & 117.95 \\
\hline lnzz & 1.2.1.3; & 9.97 & 12.97 & 30.16 \\
\hline $100 \mathrm{~s}$ & 1.1 .1 .38 & 10.64 & 15.34 & 23.67 \\
\hline $109 b$ & 1.1.1.25; & 9.98 & 15.17 & 20.21 \\
\hline 1p91 & 1.3.1.26; & 10.61 & 14.56 & -22.64 \\
\hline $1 p j 2$ & 1.1.1.39; & 8.70 & 13.00 & -5.45 \\
\hline $1 \mathrm{t} 26$ & 1.1.1.27; & 10.50 & 14.74 & -12.62 \\
\hline $1 \mathrm{t} 2 \mathrm{e}$ & 1.1.1.27; & 10.20 & 14.60 & -9.47 \\
\hline $1 v 35$ & 1.3.1.9; & 9.88 & 14.56 & 36.57 \\
\hline $1 \mathrm{vl0}$ & & 10.44 & 14.74 & -27.23 \\
\hline 1vrw & 1.3.1.9; & 10.00 & 14.58 & 33.57 \\
\hline $1 \mathrm{wnb}$ & 1.2.1.8; & 9.73 & 11.98 & 102.63 \\
\hline $1 \mathrm{yl} 7$ & 1.3.1.26; & 10.52 & 14.86 & -10.27 \\
\hline $1 z j y$ & 1.1.1.2; & 8.73 & 13.52 & -85.10 \\
\hline $1 \mathrm{zmd}$ & 1.8.1.4; & 11.85 & 17.41 & -159.78 \\
\hline $1 z p t$ & 1.7.99.5; & 6.55 & 3.77 & 49.76 \\
\hline $2 \mathrm{a} 92$ & 1.1.1.27; & 10.47 & 14.75 & -14.64 \\
\hline 2aq8 & 1.3.1.9; & 9.32 & 14.48 & 9.02 \\
\hline $2 \mathrm{aqh}$ & 1.3.1.9; & 9.49 & 14.52 & 15.80 \\
\hline 2aqi & 1.3.1.9; & 9.38 & 14.57 & 8.29 \\
\hline $2 \mathrm{dkn}$ & 1.1.1.50; & 9.59 & 13.89 & -0.67 \\
\hline 2dld & 1.1.1.28; & 10.95 & 14.68 & 21.24 \\
\hline 2dte & 1.1.1.118; & 9.84 & 14.20 & -7.17 \\
\hline $2 \mathrm{frd}$ & 1.6.1.2; & 9.93 & 16.74 & 45.07 \\
\hline $2 \mathrm{p} 9 \mathrm{c}$ & 1.1.1.95; & 10.45 & 14.73 & -19.26 \\
\hline $2 p 9 g$ & 1.1.1.95; & 10.48 & 14.63 & -21.92 \\
\hline $2 q 3 e$ & 1.1.1.22; & 11.09 & 15.12 & 29.38 \\
\hline $2 \mathrm{vhv}$ & 1.4.1.1; & 10.68 & 15.46 & 16.19 \\
\hline 2vhw & 1.4.1.1; & 10.76 & 15.59 & -3.87 \\
\hline
\end{tabular}




\begin{tabular}{|c|c|c|c|c|}
\hline $2 \mathrm{x} 0 \mathrm{i}$ & 1.1.1.37; & 10.42 & 14.94 & 8.88 \\
\hline $3 a d p$ & 1.1.1.45; & 10.83 & 14.79 & 31.2 \\
\hline $3 \mathrm{amz}$ & $1.17 .1 .4,1$ & 10.72 & 16.98 & -66.18 \\
\hline 3aut & 1.1.1.47; & 9.61 & 14.24 & -18.83 \\
\hline $3 a y 6$ & 1.1.1.47; & 9.53 & 14.18 & 5.03 \\
\hline $3 \mathrm{flk}$ & 1.1.1.93; & 10.27 & 11.64 & 104.54 \\
\hline 3 ggo & & 10.59 & 14.37 & 42.81 \\
\hline $3 \mathrm{~h} 3 \mathrm{f}$ & 1.1 .1 .27 & 10.33 & 14.95 & 3.42 \\
\hline 3iam & 1.6 .99 .5 & 11.47 & 17.94 & -101.94 \\
\hline 3iqd & 1.5.1.11; & 11.26 & 15.67 & -61.60 \\
\hline 3 meq & & 10.41 & 14.75 & -3.74 \\
\hline $3 \mathrm{mw} 9$ & 1.4.1.3 & 9.75 & 11.16 & -32.55 \\
\hline $3 n t 2$ & 1.1.1.18; & 10.75 & 14.67 & -24.04 \\
\hline $3 p l r$ & 1.1.1.22; & 11.06 & 14.53 & 41.31 \\
\hline 3 prj & 1.1.1.22; & 10.72 & 12.59 & -71.28 \\
\hline $3 q 2 i$ & & 10.91 & 15.64 & 51.81 \\
\hline $3 \mathrm{q} 2 \mathrm{k}$ & & 10.95 & 15.42 & 74.61 \\
\hline $3 \mathrm{rfv}$ & 1.1.1.203; & 9.77 & 14.62 & -6.01 \\
\hline 3uh1 & 1.5.1.7; & 10.62 & 14.80 & 23.04 \\
\hline 3uni & $1.17 .1 .4,1$ & 10.33 & 16.57 & -62.42 \\
\hline $3 z u 3$ & & 10.48 & 14.92 & 38.22 \\
\hline $4 \mathrm{bkr}$ & 1.3.1.-, 1. & 10.44 & 14.93 & 33.83 \\
\hline $4 \mathrm{bku}$ & 1.3.1.9; & 9.47 & 14.48 & 13.73 \\
\hline $4 c v 2$ & 1.3.1.9; & 9.56 & 14.61 & 12.50 \\
\hline $4 d q u$ & 1.3.1.9; & 9.44 & 14.55 & 23.91 \\
\hline $4 d t i$ & 1.3.1.9; & 9.38 & 14.59 & 0.25 \\
\hline 4eue & 1.3.1.-; & 10.29 & 14.97 & 54.20 \\
\hline $4 \mathrm{fw} 8$ & 1.1.1.100; & 10.05 & 14.28 & -64.13 \\
\hline $4 g 6 h$ & 1.6.5.9; & 10.61 & 15.74 & 143.44 \\
\hline $4 \mathrm{~g} 14$ & $1.1 .1 .1,1$ & 10.84 & 15.19 & -47.58 \\
\hline $4 \mathrm{~h} 8 \mathrm{a}$ & 1.1.1.154; & 11.25 & 17.98 & -5.30 \\
\hline 4ilk & 1.1.1.-; & 10.39 & 14.58 & -19.20 \\
\hline $4 \operatorname{im} 7$ & 1.-.-.--; & 9.62 & 13.85 & 29.30 \\
\hline 4itu & & 10.10 & 13.75 & -25.18 \\
\hline $4 \mathrm{j} 4 \mathrm{~h}$ & 1.4.1.-; & 10.64 & 15.11 & -16.48 \\
\hline 4jnk & 1.1.1.27; & 10.28 & 15.05 & -2.97 \\
\hline $41 t n$ & 1.5.1.42; & 10.23 & 12.18 & 61.49 \\
\hline $4 \mathrm{nbu}$ & & 9.59 & 13.79 & -10.80 \\
\hline 4 plt & & 10.43 & 14.84 & -3.71 \\
\hline 4plw & & 10.50 & 14.93 & -2.91 \\
\hline $4 \mathrm{plz}$ & 1.1.1.27; & 10.47 & 14.86 & -12.80 \\
\hline $4 \mathrm{q} 9 \mathrm{n}$ & 1.3.1.9; & 9.91 & 14.54 & 25.47 \\
\hline
\end{tabular}

${ }^{\mathrm{a}} \mathrm{A}$ blank in the EC column indicates the EC number is uncertain. 


\section{References}

(1) Allen, F. H. Acta Crystallogr. 2002, B58, 380-388.

(2) Dewar, M. J. S.; Zoebisch, E. G.; Healy, E. F.; Stewart, J. J. P. J. Am. Chem. Soc. 1985, 107, 3902-3909.

(3) Stewart, J. J. P. J. Mol. Mod. 2004, 10, 155-164.

(4) Dewar, M. J. S.; Thiel, W. J. Am. Chem. Soc. 1977, 99, 4899-4907.

(5) Becke, A. D. Phys. Rev. A 1988, 38, 3098-3100.

(6) Lee, C.; Yang, W.; Parr, R. G. Phys. Rev. B 1988, 37, 785-789.

(7) Jorgensen, W. L.; Chandrasekhar, J.; Madura, J. D.; Impey, R. W .; Klein, M. L. J. Chem. Phys. 1983, 79, 926-935.

(8) MacKerell, J. A. D.; Bashford, D.; Bellott, M.; Dunbrack, R.; Evanseck, J. D.; Field, M. J.; Fischer, S.; Gao, J.; Guo, H.; Ha, S.; Joseph-McCarthy, D.; Kuchnir, L.; Kuczera, K.; Lau, F. T. K.; Mattos, C.; Michnick, S.; Ngo, T.; Nguyen, D. T.; Prodhom, B.; Reiher, W. E. I.; Roux, B.; Schlenkrich, M.; Smith, J. C.; Stote, R.; Straub, J.; Watanabe, M.; Wiorkiewicz-Kuczera, J.; Yin, D.; Karplus, M. J. Phys. Chem. B. 1998, 102, 3586-3616.

(9) Ryckaert, J. P.; Ciccotti, G.; Berendsen, H. J. C. J. Comput. Phys. 1977, 23, 327-341.

(10) Allen, M. P.; Tildesley, D. J. Computer Simulation of Liquids; Oxford University Press: NY, 1987.

(11) Brooks, B. R.; Brooks III, C. L.; Mackerell, A. D.; Nilsson, L.; Petrella, R. J.; Roux, B.; Won, Y.; Archontis, G.; Bartels, C.; Boresch, S.; Caflisch, A.; Caves, L.; Cui, Q.; Dinner, A. R.; Feig, M.; Fischer, S.; Gao, J.; Hodoscek, M.; Im, W.; Kuczera, K.; Lazaridis, T.; Ma, J.; Ovchinnikov, V.; Paci, E.; Pastor, R. W.; Post, C. B.; Pu, J. Z.; Schaefer, M.; Tidor, B.; Venable, R. M.; Woodcock, H. L.; Wu, X.; Yang, W.; York, D. M.; Karplus, M. J. Comput. Chem. 2009, 30, 1545-1615.

(12) Kumar, S.; Rosenberg, J. M.; Bouzida, D.; Swendsen, R. H.; Kollman, P. A. J. Comput. Chem. 1992, 13, 1011-1021.

(13) Frisch, M. J.; Trucks, G. W.; Schlegel, H. B.; Scuseria, G. E.; Robb, M. A.; Cheeseman, J. R.; Scalmani, G.; Barone, V.; Mennucci, B.; Petersson, G. A.; Nakatsuji, H.; Caricato, M.; Li, X.; Hratchian, H. P.; Izmaylov, A. F.; Bloino, J.; Zheng, G.; Sonnenberg, J. L.; Hada, M.; Ehara, M.; Toyota, K.; Fukuda, R.; Hasegawa, J.; Ishida, M.; Nakajima, T.; Honda, Y.; Kitao, O.; Nakai, H.; Vreven, T.; Montgomery, J. A., Jr.; Peralta, J. E.; Ogliaro, F.; Bearpark, M.; Heyd, J. J.; Brothers, E.; Kudin, K. N.; Staroverov, V. N.; Kobayashi, R.; Normand, J.; Raghavachari, K.; Rendell, A.; Burant, J. C.; Iyengar, S. S.; Tomasi, J.; Cossi, M.; Rega, N.; Millam, J. M.; Klene, M.; Knox, J. E.; Cross, J. B.; Bakken, V.; Adamo, C.; Jaramillo, J.; Gomperts, R.; Stratmann, R. E.; Yazyev, O.; Austin, A. J.; Cammi, R.; Pomelli, C.; Ochterski, J. W.; Martin, R. L.; Morokuma, K.; Zakrzewski, V. G.; Voth, G. A.; Salvador, P.; Dannenberg, J. J.; Dapprich, S.; Daniels, A. D.; Farkas, O.; Foresman, J. B.; Ortiz, J. V.; Cioslowski, J.; Fox, D. J.; Gaussian, Inc.: Wallingford CT, 2009.

(14) Hua, Y. H.; Wu, C. Y.; Sargsyan, K.; Lim, C. Sci. Rep. 2014, 4, 6471.

(15) Berman, H. M.; Battistuz, T.; Bhat, T. N.; Bluhm, W. F.; Bourne, P. E.; Burkhardt, K.; Feng, Z.; Gilliland, G. L.; Iype, L.; Jain, S.; Fagan, P.; Marvin, J.; Padilla, D.; Ravichandran, V.; Schneider, B.; Thanki, N.; Weissig, H.; Westbrook, J. D.; Zardecki, C. Acta crystallogr. Sect. D-Biol. Crystallogr. 2002, 58, 899-907. 